ORIGINAL ARTICLE

\title{
Serum Proinsulin in Bangladeshi Subjects with Impaired Glucose Tolerance
}

\author{
S Sultana ${ }^{1}$, Z Zeba $^{2}$, A Hossain ${ }^{2}$, A Khaleque ${ }^{1}$, R Zinnat ${ }^{2}$, L Ali $^{2}$ \\ ${ }^{1}$ Dept of Life Sciences, North South University, Dhaka \\ ${ }^{2}$ Dept of Biochemistry and Cell Biology, Bangladesh University of HealthSciences (BUHS), Dhaka
}

\begin{abstract}
Hyperproinsulinemia is commonly present in subjects with impaired glucose tolerance. The present study was undertaken to investigate the proinsulin level in Bangladeshi IGT subjects and to explore its association with insulin resistance. This observational study was conducted under a case-control design with IGT subjects $(n=50)$ and controls $(n=44)$. IGT was diagnosed following the WHO Study Group Criteria. Serum glucose was measured by glucose-oxidase method, serum lipid profile by enzymatic method and serum insulin and serum proinsulin were measured by ELISA method. Insulin secretory capacity (HOMA\%B) and insulin sensitivity (HOMA\%S) were calculated from fasting serum glucose and fasting serum insulin by homeostasis model assessment. The study subjects were age- and BMI- matched. Mean $( \pm$ SD) age (yrs) of the control and IGT subjects were $40 \pm 6$ and $40 \pm 5$ respectively $(\mathrm{p}=0.853)$. Mean $( \pm \mathrm{SD})$ BMI of the control and IGT subjects were $23 \pm 3$ and $22 \pm 2$ respectively $(p=0.123)$. Fasting glucose was not significantly higher in IGT subjects, but serum glucose 2 hours after $75 \mathrm{gm}$ glucose load was significantly higher in IGT subjects. Median (Range) value of fasting serum glucose $(\mathrm{mmol} / \mathrm{l})$ of control and IGT subjects were $5.3(3.8-6)$ and 5.2 (4-12) respectively; $(\mathrm{p}=0.297)$. Median (Range) value of serum glucose (mmol/l) 2 hours after $75 \mathrm{gm}$ glucose load of control and IGT subjects were 6.1 (3-7.8) and 7.9 (521) respectively; $(p=0.001)$. Fasting TG was significantly higher in IGT subjects and LDL-c was significantly lower in IGT subjects. Serum Total cholesterol and HDL-c were not significantly different between the IGT and control subjects. Median (Range) value of fasting serum TG (mg/dl) of control and IGT subjects were $119(51-474)$ and $178(82-540)$ respectively; $(\mathrm{p}=0.001)$. Median (Range) value of fasting serum T chol (mg/dl) of control and IGT subjects were $180(65-272)$ and 186 (140-400) respectively; $(\mathrm{p}=0.191)$. Median (Range) value of fasting serum HDL-C (mg/dl) of control and IGT subjects were 29 (19-45) and 31 (15-78) respectively; $(\mathrm{p}=0.914)$. Median (Range) value of fasting serum LDL-C (mg/dl) of control and IGT subjects were 117(29-201) and 111(41$320)$ respectively; $(p=0.001)$. Fasting serum proinsulin was significantly higher in IGT subjects. Median (Range) value of fasting serum proinsulin (pmol/l) of control and IGT subjects were 9.2(1.8-156) and 17(3-51) respectively; $(\mathrm{p}=0.001)$. Insulin secretory capacity (HOMA\%B) was higher but insulin sensitivity (HOMA\%S) was significantly lower in case of IGT subjects. Median (Range) value of HOMA\%B of control and IGT subjects were 97(46-498) and 164(17-300) respectively; $(\mathrm{p}=0.001)$. Median (Range) value of HOMA\%S of control and IGT subjects were $68(19-270)$ and $39(15-110)$ respectively $(p=0.001)$. In multiple regression analysis a significant negative association was found between fasting proinsulin and insulin sensitivity $(p=0.037)$. The data led to the following conclusions: a) Insulin resistance is the predominant defect in Bangladeshi IGT subjects. b) Basal proinsulin level is significantly increased in IGT subjects. c) Insulin resistance is negatively associated with serum proinsulin in IGT subjects.

Key Words: Proinsulin, Hyperproinsulinemia, IGT, Insulin Resistance
\end{abstract}

\section{Introduction}

Plasma concentration of proinsulin is raised in subjects with Type II (non-insulin-dependent)

diabetes mellitus ${ }^{1}$. Proinsulin is normally converted within the beta-cell into split proinsulin

Bangladesh J Med Biochem 2014; 7(2): 41-46 

and subsequently into insulin and C-peptide ${ }^{2}$. In subjects with impaired glucose tolerance (IGT), the relative (to insulin) proinsulin and absolute proinsulin concentrations are important predictors of progression to Type II diabetes ${ }^{3,4}$. The evaluation of proinsulin concentration in populations at risk of diabetes can be complicated by several factors, including insulin resistance, obesity, and familial predisposition to diabetes. For example, although established type 2 diabetes is associated with hyperproinsulinemia, it has been suggested that the normal $\beta$-cell response to increased secretory demand is due to enhanced efficiency of proinsulin processing, leading to a decreased proinsulin/insulin ratio. In support of this concept, both insulin resistance and its clinical correlate, obesity, have been shown to be associated with decreased proinsulin/insulin ratio in normoglycemic subjects ${ }^{5-7}$.

The relationship between elevated proinsulin levels and glucose intolerance or the risk of T2DM may have several explanations ${ }^{8}$. Increased secretion of proinsulin could be secondary to external factors acting on the $\beta$-cell, an inherent abnormality of the $\beta$-cell itself, or a combination of both. Hyperglycemia in subjects with IGT and T2DM might be associated with an increase in the proportion of proinsulin relative to insulin secreted by the $\beta$-cell. In addition, subjects with IGT and T2DM, as well as offspring of T2DM patients, are often insulin resistant. The increased $\beta$-cell secretory demand due to insulin resistance could result in disproportionate changes in plasma proinsulin levels. Finally, a disproportionate increase in plasma proinsulin levels could be a marker of $\beta$ cell distress or compromised insulin secretion. Previous data on the association of proinsulin levels with insulin resistance and $\beta$-cell function have come mainly from experimental studies. It is evident that insulin resistance in normoglycemic subjects was related to decreased proinsulin relative to insulin levels. This suggests that insulin resistance is not a trigger for disproportionately increased proinsulin secretion.

It shows that relative hyperproinsulinemia is associated with a delayed insulin response to glucose in a homogeneous impaired glucose

tolerant study group. In previous studies no association was found between relative hyperproinsulinaemia and insulin sensitivity, in contrast to another study ${ }^{6}$ in which a negative association was found in 138 people with normal glucose tolerance.

Bangladesh has one of the largest diabetic and prediabetic populations in the world, but the pathophysiology of these states in our population has only been started to be investigated. Data from this population shows that both insulin secretory defect and insulin resistance are present in T2DM subjects, but the secretory defect seems to have a predominant role ${ }^{9,10}$. In case of prediabetes, studies from Bangladeshi population, it was reported that IFG and IGT seemed to be separate disorders where $\beta$-cell dysfunction was predominant in IFG and insulin resistance had a major role in IGT. A combined IFG-IGT group has both the defects ${ }^{11}$.

It has been found that the lean and young Bangladeshi diabetic population showed disproportionate rise of proinsulin compared to the healthy counterparts ${ }^{12}$. No study has yet been done to investigate the role of serum proinsulin in the IGT subjects. Studies on prediabetic subjects can give substantial insight on the natural history of the disorders and the present study was undertaken to investigate the association of proinsulin level with insulin resistance in IGT subjects.

\section{Materials and Methods}

Ninety four subjects were taken into the study among them 50 were IGT subjects (WHO guidelines) and 44 were control subjects. The study subjects were adult, age ranging from 2555 years and voluntarily agreed to participate in the study. Patients having any co-morbid diseases and pregnant women were excluded from the study.

Anthropometric measurements such as BMI, percentages of body fat mass (by Omeron body fat monitor, Japan), blood pressure (according to WHO-ISH) were recorded for all study subjects. 
Venous blood were taken for biochemical and Glycemic and lipidemic status of the study serological analysis such as serum glucose (by subjects

Glucose Oxidase, GOD-PAP method), Total cholesterol (by enzymatic endpoint, Cholesterol Oxidase/peroxidase method), TG (by enzymatic colorimetric, GPO-PAP method), HDL cholesterol (by enzymatic colorimetric, CHOD-PAP method), LDL cholesterol (by using Friedewald's formula), Creatinine (by alkaline picrate, GPO-PAP method), SGPT (by UV method) and serum insulin and proinsulin were estimated by ELISA method.

\section{Results}

A total number of 94 subjects were enrolled in this study among them 50 were isolated IGT (WHO guidelines) and 44 were control subjects without any family history of DM and IGR (Impaired glucose regulation).

Anthropometric and clinical characteristics of the study subjects

The study subjects were age and BMI matched. Mean $( \pm \mathrm{SD})$ age (yrs) of the control and IGT subjects were $40 \pm 6$ and $40 \pm 5$ respectively $(\mathrm{p}=0.853)$. Mean $( \pm \mathrm{SD}) \mathrm{BMI}$ of the control and IGT subjects were $23 \pm 3$ and $22 \pm 2$ respectively $(\mathrm{p}=0.123)$. Both SBP and DBP did not differ between groups as shown in table I.

Table-I: Anthropometric and clinical haracteristics of the study subject

\begin{tabular}{lccc}
\hline Variable & Control $(\mathbf{n = 4 4 )}$ & IGT $(\mathbf{n}=\mathbf{5 0})$ & $\mathbf{t} / \mathbf{p}$ values \\
\hline Age (years) & $40 \pm 6$ & $40 \pm 5$ & $0.186 / 0.853$ \\
BMI (kg/m) & $23 \pm 3$ & $22 \pm 2$ & $1.55 / 0.123$ \\
SBP (mm Hg) & $108 \pm 8$ & $118 \pm 15$ & $1.680 / 0.096$ \\
DBP (mm Hg) & $71 \pm 12$ & $72 \pm 9$ & $1.053 / 0.397$
\end{tabular}

Results are expressed as mean SD. Significance of difference was calculated by unpaired student's $t$ tests at 5\% significance level. $n=$ numbers of subjects; IGT, impaired glucose tolerance; BMI, Body mass index; SBP, Systolic blood pressure; DBP, Diastolic blood pressure.
Fasting glucose was not significantly higher in IGT subjects but serum glucose 2 hours after 75 gm glucose load was significantly higher in IGT subjects. Median (Range) value of fasting serum glucose $(\mathrm{mmol} / \mathrm{l})$ of control and IGT subjects were $5.3(3.8-6)$ and 5.2 (4-12) respectively; $(p=0.297)$. Median (Range) value of serum glucose $(\mathrm{mmol} / \mathrm{l}) 2$ hours after 75 gm glucose load of control and IGT subjects were 6.1 (3$7.8)$ and $7.9(5-21)$ respectively; $(p=0.001)$.

Serum Total cholesterol and HDL-c were not significantly different between the IGT and control subjects. Fasting TG was significantly higher in IGT subjects and LDL-c was significantly lower in IGT subjects. Median (Range) value of fasting serum TG $(\mathrm{mg} / \mathrm{dl})$ of control and IGT subjects were 119 (51-474) and $178(82-540)$ respectively; $(p=0.001)$. Median (Range) value of fasting serum $\mathrm{T}$. chol $(\mathrm{mg} / \mathrm{dl})$ of control and IGT subjects were 180 (65-272) and 186 $(140-400)$ respectively; $(p=0.191)$. Median (Range) value of fasting serum HDL-C $(\mathrm{mg} / \mathrm{dl})$ of control and IGT subjects were 29 (19-45) and 31 (15-78) respectively; $(p=0.914)$. Median (Range) value of fasting serum LDL-C $(\mathrm{mg} / \mathrm{dl})$ of control and IGT subjects were 117(29-201) and 111(41-320) respectively; $(\mathrm{p}=0.001)$ as shown in table II.

Table-II: Glycemic and lipidemic status of the study subjects

\begin{tabular}{llll}
\hline Variable & Control $(\mathbf{n}=\mathbf{4 4})$ & IGT $(\mathbf{n = 5 0 )}$ & $\mathbf{t} / \mathbf{p}$ values \\
\hline F_Glu (mmol/l) & $5.3(3.8-6)$ & $5.2(4-12)$ & $1.043 / 0.297$ \\
2h_Glu (mmol/l) & $6.1(3-7.8)$ & $7.9(5-21)$ & $5.912 / 0.001$ \\
TG (mg/dl) & $119(51-474)$ & $178(82-540)$ & $4.107 / 0.001$ \\
T Chol (mg/dl) & $180(65-272)$ & $186(140-400)$ & $1.308 / 0.191$ \\
HDL-C (mg/dl) & $29(19-45)$ & $31(15-78)$ & $1.043 / 0.297$ \\
LDL-C (mg/dl) & $117(29-201)$ & $111(41-320)$ & $0.572 / 0.567$
\end{tabular}

Results are expressed as median (range). Significance of difference was calculated by Mann -Whitney U test at 5\% significance level. $n=$ numbers of subjects; IGT, impaired glucose tolerance; $F$ Glu, Fasting glucose; 2h_Glu, 2 hour after glucose; $T$ chol, Total cholesterol; TG, Triglycerides; $\quad H D L-C$, high density lipoprotien cholesterol; $L D L-C$, low density lipoprotien cholesterol. 
Serum Proinsulin in Bangladeshi Subjects with Impaired Glucose Tolerance

Insulin sensitivity, B-cell function and proinsulinemic status of the study subjects

Fasting serum proinsulin was significantly higher in IGT subjects. Median (Range) value of fasting serum proinsulin (pmol/l) of control and IGT subjects were 9.2(1.8-156) and 17 (3-51 respectively); $(\mathrm{p}=0.001)$. Insulin secretory capacity (HOMA\% B) was higher but insulin sensitivity (HOMA\%S) was significantly lower in case of IGT subjects. Median (Range) value of HOMA\%B of control and IGT subjects were 97 (46-498) and $164(17-300$ respectively $) ; \quad(p=0.001)$. Median (Range) value of HOMA\%S of control and IGT subjects were 68(19-270) and 39(15-110) respectively $(\mathrm{p}=0.001)$ as shown in table III.

Table III: Insulin sensitivity, ß-cell function and proinsulinemic status of the study subjects

\begin{tabular}{llll}
\hline Variable & Control $(\mathrm{n}=44)$ & IGT $(\mathrm{n}=50)$ & t/p values \\
\hline HOMA\%B & $97(46-498)$ & $164(17-300)$ & $4.463 / 0.001$ \\
HOMA\%S & $68(19-270)$ & $39(15-110)$ & $4.512 / 0.001$ \\
$\begin{array}{l}\text { Fasting Serum } \\
\text { Proinsulin (pmol/L) }\end{array}$ & $9.2(1.8-156)$ & $17(3-51)$ & $3.470 / 0.001$ \\
\hline
\end{tabular}

Results are expressed median (range). Significance of difference was calculated by Mann -Whitney U test at 5\% significance level. $n=$ numbers of subjects; IGT, impaired glucose tolerance; fasting glucose; HOMA\%B, $\beta$-cell function assessed by homeostasis model assessment; HOMA\%S, insulin sensitivity assessed by homeostasis model assessed, F S Proinsulin, Fasting serum proinsulin.

\section{Regression Analysis}

In the multiple regression analysis taking insulin secretory defect as dependent variable and age, BMI, proinsulin and control/IGT as independent variables no significant association was found (Table IV).

Table IV: Multiple regression analysis taking HOMA\% B as a dependent variable in IGT subjects

\begin{tabular}{lcl}
\hline Variable & $\beta$ value & P value \\
\hline Age & 0.076 & 0.505 \\
BMI & 0.024 & 0.836 \\
Proinsulin & 0.037 & 0.755 \\
Group (Control/IGT) & -0.095 & 0.406 \\
\hline
\end{tabular}

Multiple regression analysis was done taking $\mathrm{HOMA \% B}$ as a dependent variable and Group (Control/IGT), Age, BMI as independent co variables.
In multiple regression analysis a significant negative association was found between fasting proinsulin and insulin sensitivity $(\mathrm{p}=0.037)$ as shown in table- $\mathrm{V}$.

Table V: Multiple regression analysis taking $\mathrm{HOMA} \% \mathrm{~S}$ as a dependent variable in IGT subjects

\begin{tabular}{lcl}
\hline Variable & value & P value \\
\hline BMI & $\beta-0.141$ & 0.183 \\
Proinsulin & -0.228 & 0.037 \\
Group (Control/IGT) & 0.197 & 0.065 \\
\hline
\end{tabular}

Multiple regression analysis was done taking HOMA\%S as a dependent variable and Group (Control/IGT), BMI, as independent co variables. HOMA\%S had significant negative association $(p=0.037)$ with proinsulin.

\section{Discussion}

Analysis of the anthropometric data in the present study showed that the IGT and the Control subjects were age and BMI matched. Both the IGT and Controls were in the middle age group (mean age was 40) as shown in table I. Obesity is one of the major risk factors for the development of IGT, but the IGT subjects in the present study did not show any generalized obesity and their BMI were within normal range. Regarding the lipid profile, IGT subjects had significantly higher serum triglyceride level than that of controls. Serum LDL cholesterol was found to be higher both in controls and IGT subjects but difference between controls and the IGT were not significant. This finding is consistent with the finding of a study where it was found that people with IGT had higher fasting plasma triglycerides and non-esterified fatty acids, and lower HDL cholesterol concentrations than people with NGT and with IFG. Blood lipid profiles illustrate that even non-obese people with IGT show several features of metabolic syndrome ${ }^{13}$.

Insulin secretory defect was not evident in IGT subjects rather a hypersecretory stage was found by increased $\beta$-cell function evident by HOMA\% B. Wasada et. al also found similar findings and they found that people with IGT 
showed a compensatory increase in insulin secretion as well as manifestations of metabolic syndrome ${ }^{13}$.

IGT subjects had significantly lower insulin sensitivity $(p=0.0001)$ compared to Controls (Table III) which indicates that IGT subjects had insulin resistance. This finding is consistent with the previous studies done in our IGT population where insulin resistance was found as a primary defect for IGT subjects. Data from the Bangladeshi population indicate that IFG has primarily insulin secretory defect, IGT has primarily insulin resistance, and combined IFGIGT has both the defects ${ }^{11}$. Another study in western population showed that insulin resistance is markedly higher in people with isolated IGT and is absent in people with IFG $^{14}$. In contrast, some studies have reported that people with isolated IFG are more insulin resistant than those with normal glucose tolerance (NGT) and people with isolated IGT exhibited a more severe deficit in insulin secretion than those with $\mathrm{IFG}^{15,16}$. Although both isolated IFG and isolated IGT are insulinresistant states, they differ in their site of insulin resistance $^{17}$. People with isolated IFG predominantly have hepatic insulin resistance and normal muscle insulin sensitivity, whereas individuals with isolated IGT have normal to slightly reduced hepatic insulin sensitivity and moderate to severe muscle insulin resistance. Not surprisingly, individuals with both IFG and IGT manifest both muscle and hepatic insulin resistance.

In the present study, the basal plasma proinsulin (pmol/L) level was $9.2(1.8-156)$ in control group and 17 (3-51) in IGT. This value is slightly higher compared to the value found in western population where they got $5.8 \pm 3.3$ $\mathrm{pmol} / \mathrm{l}$ in control group, $12.6 \pm 7.5 \mathrm{pmol} / 1$ in IGT group $^{1}$.

Stumvoll et al. found a contrasting findings and they suggested that the primary lesion resulting in the secretory defect of IGT subjects did not necessarily involve abnormal proinsulin processing ${ }^{18}$. If this had been the case, more proinsulin per mol insulin should have been cosecreted, resulting in an even greater difference between the two groups during maximal stimulation. It is possible that the activity of the specific endopeptidases and exopeptidases responsible for the enzymatic conversion of proinsulin to insulin increased over proportionately in IGT during maximal stimulation. This could indicate that a defect in the rate of conversion of proinsulin to insulin is overcome when the activity of the $\beta$-cell is strongly stimulated ${ }^{18}$.

In the multiple regression analysis taking insulin secretory defect as dependent variable and age, BMI, proinsulin and control/IGT as independent variables no significant association was found (Table IV).

In the multiple regression analysis taking insulin sensitivity as dependent variable and BMI, proinsulin and control/IGT as independent variables a significant negative association was found with serum proinsulin $(\mathrm{p}=0.05)$ as shown in table V. Lele et al. also found elevated proinsulin and its association with insulin resistance in Indian population ${ }^{19}$. But Wang et al. did not found any such association and they found that, the fasting proinsulin to insulin ratio showed no relationship to the degree of insulin resistance. They suggested that, insulin resistance and the need to secrete more insulin to maintain glucose tolerance did not necessarily lead to abnormal insulin processing by the $\beta$ cell $^{20}$. From this study, it can be concluded that insulin resistance is the predominant defect in Bangadeshi IGT subjects and basal proinsulin level is significantly increased in IGT subjects while insulin resistance is negatively associated with serum proinsulin in IGT subjects.

\section{References}

1. Yoshioka N, Kuzuya T, Matsuda A, Taniguchi M, Iwamoto Y. Serum proinsulin concentrations at fasting and after oral glucose load in patients with Type II (non-insulin-dependent) diabetes mellitus. Diabetologia 1988; 31: 355-360.

2. Rhodes CJ, Alarcon C. What $\beta$-cell defect could lead to hyperproinsulinaemia in NIDDM? Some clues from recent advances made in understanding the proinsulin-processing mechanism. Diabetes 1994; 43: 511-517. 
Serum Proinsulin in Bangladeshi Subjects with Impaired Glucose Tolerance

3. Haffner SM, Gonzalez C, Mykkanen L, Stern M. Total immunoreactive proinsulin, immunoreactive insulin and specific insulin in relation to conversion to NIDDM: the Mexico City Diabetes Study. Diabetologia 1997; 40: 830-837.

4. Nijpels G, Popp-Snijders C, Kostense PJ, Bouter LM, Heine RJ. Fasting proinsulin and 2-h post-load glucose concentrations predict the conversion to NIDDM in subjects with impaired glucose tolerance: the Hoorn Study. Diabetologia 1996; 39: 113-118

5. Haffner SM, Stern MP, Miettinen H, Gingerich R, Bowsher RR. Higher proinsulin and specific insulin are both associated with a parental history of diabetes in nondiabetic Mexican-American subjects. Diabetes 1995; 44:1156-1160.

6. Mykkanen L, Haffner SM, Hales CN, Ronnemaa T, Laakso M: The relation of proinsulin, insulin, and proinsulin-to-insulin ratio to insulin sensitivity and acute insulin response in normoglycemic subjects. Diabetes 1997; 46:1990-1995.

7. Roder ME, Dinesen B, Hartling SG, Houssa P, Vestergaard H, Sodoyez-Goffaux F, Binder C. Intact proinsulin and $\beta$-cell function in lean and obese subjects with and without type 2 diabetes. Diabetes Care 1999; 22: 609-614.

8. Hales $\mathrm{CN}$, Byrne CD, Petry CJ, Wareham NJ: Measurement of insulin and proinsulin. Diabetes Rev 1996; 4: 320-335.

9. Roy MN, Biswas KB, Siddique N, Arslan MI, Ali L. Determinants of insulin secretion sensitivity in Bangladeshi type 2 diabetic subjects. Metab Syndr Relat Disord 2007; 5: 275-281.

10. Zinnat R. Role of insulin deficiency and insulin resistance in the pathogenesis of type 2 diabetes in young Bangladeshi subjects. 1999; M Phil Thesis (Medical Biochemistry), University of Dhaka.

11. Rahman MH, Hafizur RM, Nahar Q, Khan AR, Ali L. Insulin secretion and sensitivity in Bangladeshi prediabetic subjects. J Diabetes Complications 2008; 24: 37-42.

12. Biswas SK. Role of Proinsulin and its Conversion Intermediates in the Pathogenesis of Young Onset Diabetes in Bangladesh. 2007; MPhil Thesis, Dhaka University.
13. Wasada T, Kuroki H, Arii H, Sato A, Aoki K. Who are more insulin resistant, people with IFG or people with IGT? Published online: 2004; 13 February ${ }^{\circledR}$ Springer-Verlag.

14. Carnevale Schianca GP, Rossi A, Sainaghi PP, Maduli E, Bartoli E. The significance of impaired fasting glucose versus impaired glucose tolerance: Importance of insulin secretion and resistance. Diabetes Care 2003; 26: 1333-1337

15. Tripathy $\mathrm{D}$, Carlsson $\mathrm{M}$, Almgren $\mathrm{P}$, Isomaa $\mathrm{B}$, Taskinen MR, Tuomi $\mathrm{T}$, Groop LC. Insulin secretion and insulin sensitivity in relation to glucose tolerance: lessons from the Botnia Study. Diabetes 2000; 49: 975-980.

16. Hanefeld M, Koehler C, Fuecker K, Henkel E, Schaper F, Temelkova-Kurktschiev T. Impaired Glucose Tolerance for Atherosclerosis and Diabetes study. Insulin secretion and insulin sensitivity pattern is different in isolated impaired glucose tolerance and impaired fasting glucose: the risk factor in Impaired Glucose Tolerance for Atherosclerosis and Diabetes study. Diabetes Care 2003; 26: 868-874.

17. Abdul-Ghani MA, Jenkinson CP. Insulin secretion and action in subjects with impaired fasting glucose and impaired glucose tolerance: results from the Veterans Administration Genetic Epidemiology Study. Diabetes 2006; 55: 1430-5.

18. Stumvoll M, Fritsche A, Stefan N, Hardt E, Haring $\mathrm{H}$. Evidence against a rate-limiting role of proinsulin processing for maximal insulin secretion in subjects with impaired glucose tolerance and ?cell dysfunction. J Clin Endocrinal Metab 2001; 86: $1235-1239$.

19. Lele RD, Joshi SR, Gupte A. Association of Adipocytokines (Leptin, Adiponectin TNF-alpha), insulin and proinsulin with diabetes - The Mumbai Obesity Project [MOP]. JAPI 2006; 54: 689-696.

20. Wang PW, Abbasi F, Carantoni M, Chen YD, Azhar S, Reaven GM. Insulin resistance does not change the ratio of proinsulin to insulin in normal volunteers. J Clin Endocrinol Metab 1997; 82: 3221-4. 